\title{
Tuberculosis of the extra-axial skeleton in paediatric patients
}

\author{
Sravya Vajapey ${ }^{1}$ (D), Anria Horn ${ }^{2 *}$ (iD \\ 1 Department of Orthopaedic Surgery, The Ohio State University, Columbus, Ohio, United States of America \\ 2 Department of Orthopaedic Surgery, University of Cape Town, Cape Town, South Africa \\ *anria.horn@uct.ac.za
}

Citation: Vajapey S, Horn A. Tuberculosis of the extra-axial skeleton in paediatric patients. SA Orthop J 2021;20(1):21-26. http://dx.doi.org/10.17159/23098309/2021/v20n1a2

Editor: Prof. Jacques du Toit, Stellenbosch University, Cape Town, South Africa

Received: March 2020

Accepted: October 2020

Published: March 2021

Copyright: ( 2021 Vajapey S. This is an open-access article distributed under the terms of the Creative Commons Attribution Licence, which permits unrestricted use, distribution and reproduction in any medium, provided the original author and source are credited.

Funding: No funding was received for this study.

Conflict of interest: The authors declare they have no conflicts of interest that are directly or indirectly related to the research.

\section{Abstract \\ Background}

Musculoskeletal tuberculosis (MSK TB) is a disease entity that often mimics other orthopaedic conditions in its radiographic and clinical presentation, which can delay diagnosis and treatment. The purpose of this study is to examine the clinical and radiographic presentation as well as the accuracy of various diagnostic tests, treatment, complications and outcome in paediatric patients diagnosed with MSK TB. We aim to provide insight into typical presenting features in order to expedite diagnosis in this perplexing disease.

\section{Methods}

We retrospectively reviewed 77 consecutive patients with extra-axial MSK TB treated at our institution over a ten-year period from 2008 to 2018. We collected data on initial clinical presentation, laboratory values, radiographic findings, diagnostic testing, treatment and outcomes. We performed quantitative and qualitative analysis to look for patterns in presentation that can help with diagnosis and factors affecting the clinical outcomes.

\section{Results}

The most common clinical presentation was pain of the affected limb. Constitutional symptoms were uncommon. Our patients presented with thrombocytosis and anaemia, but normal white cell counts. Inflammatory markers were mildly elevated. Of diagnostic tests employed, the Mantoux skin test yielded the most positive results $(70 \%)$ followed by tissue PCR $(53 \%)$. The hip was most frequently involved, followed by the knee and elbow. Most patients presented with normal appearing X-rays. We had a medical compliance rate of $94 \%$ with all patients followed up to completion of treatment having resolution of active disease. Thirty-nine per cent of our patients had residual joint stiffness or deformity following completion of treatment, ranging from ankylosis to mildly decreased joint range.

\section{Conclusion}

Patients with MSK TB usually present with non-specific symptoms and signs, and a high index of suspicion should be maintained in endemic areas. Typical haematological findings are an elevated ESR and CRP accompanied by anaemia and thrombocytosis. Radiographs at presentation are non-specific in more than $50 \%$ of patients. A combination of diagnostic modalities should be employed as no single test is $100 \%$ sensitive or specific. Compliance with medical treatment reliably leads to resolution of the disease. Residual joint pathology is common and needs to be addressed secondarily.

\section{Level of evidence: Level 4}

Keywords: musculoskeletal tuberculosis, paediatric tuberculosis, joints, anti-tuberculosis treatment, deformity, GeneXpert 

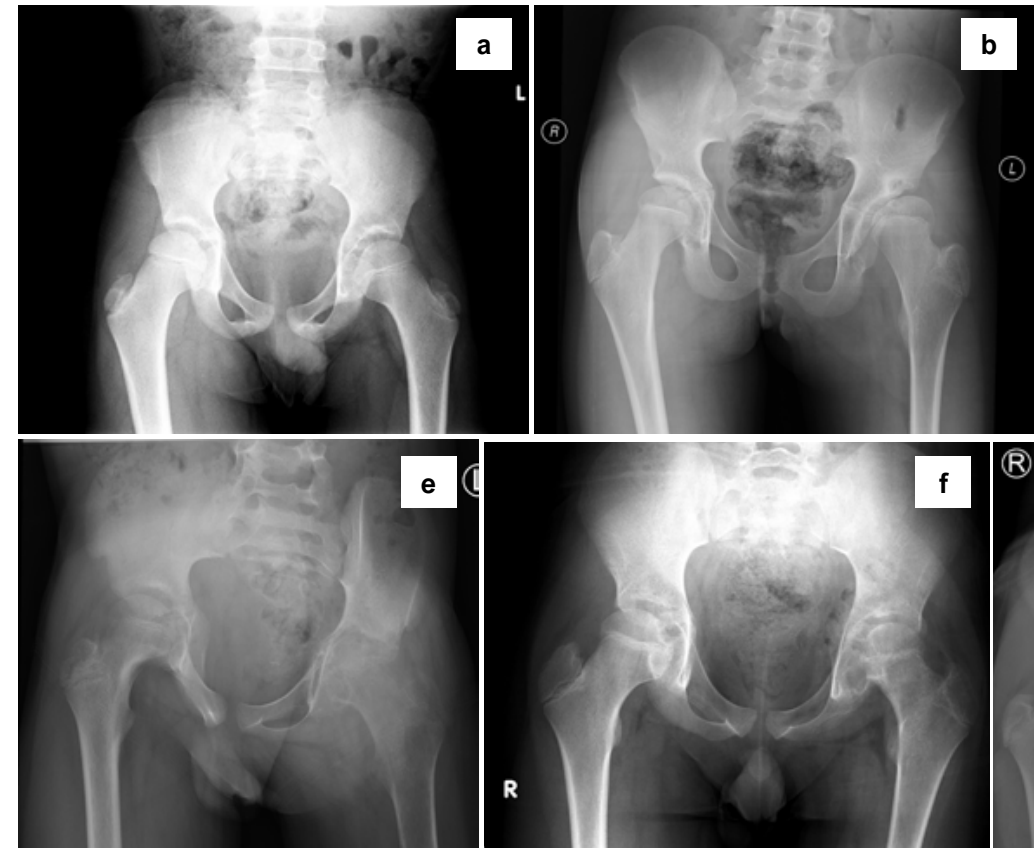

\section{Introduction}

Musculoskeletal tuberculosis (MSK TB) is a disease entity that often mimics other orthopaedic conditions in its radiographic and clinical presentation, which can delay diagnosis and treatment. While MSK TB accounts for $9.7 \%$ of all extra-pulmonary cases in the United States, this percentage is much higher in endemic regions like India and South Africa. ${ }^{1}$ Approximately 20 children each year are diagnosed with and treated for MSK TB at our institution. ${ }^{2}$

Fifty per cent of MSK TB involves the extra-axial skeleton in the form of tuberculous arthritis, osteomyelitis, bursitis, subcutaneous abscess or tenosynovitis. ${ }^{3,4}$ Diagnosing MSK TB in children is particularly challenging due to vague presenting symptoms like joint pain, fatigue and history of minor trauma, which can often obscure the underlying pathology. ${ }^{5}$

The purpose of this study is to examine the initial presentation of patients diagnosed with MSK TB to determine the characteristic clinical and radiographic features of this disease, as an aid to expedient diagnosis. We also examine the accuracy and value of the various diagnostic tests utilised, the medical and surgical treatment offered and the outcome of treatment.

\section{Materials and methods}

A retrospective review was performed on records and radiographs of all patients diagnosed with extra-axial MSK TB from January 2008 to September 2018. Approval was obtained from the institutional Human Research Ethics Committee.

We included all patients aged 14 years or less with laboratory confirmed diagnosis of MSK TB. We included five patients that were lost to follow-up or defaulted treatment, in order to assess their presenting and diagnostic features.

There were 77 patients of whom 38 were males. Mean age at presentation was 6.7 years ( 5 months to 14 years) and mean follow-up was 17 months (0-38 months).

Data was collected on demographic variables, clinical presentation, special investigations and radiological features, treatment compliance and clinical outcome. Routine investigations performed were HIV testing, full blood count (FBC), C-reactive protein (CRP) and erythrocyte sedimentation rate (ESR). Standard diagnostic tests performed were the Mantoux skin test, microscopy, culture
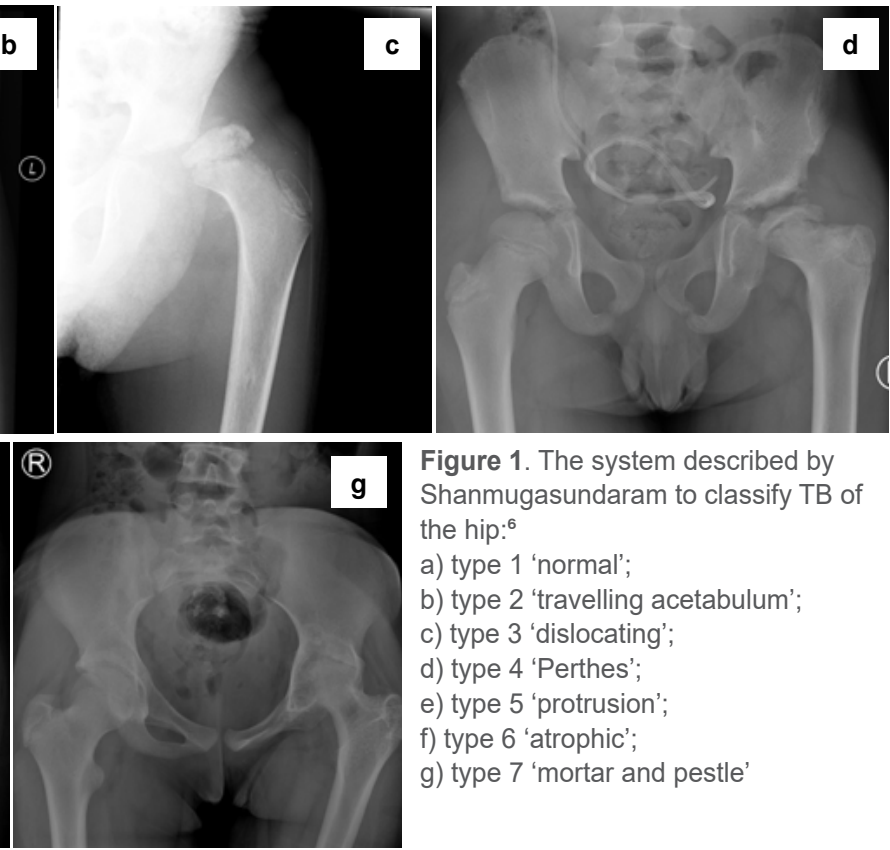

Figure 1. The system described by Shanmugasundaram to classify TB of the hip: ${ }^{6}$ a) type 1 'normal';

b) type 2 'travelling acetabulum';

c) type 3 'dislocating';

d) type 4 'Perthes';

e) type 5 'protrusion';

f) type 6 'atrophic';

g) type 7 'mortar and pestle'

and sensitivity (MCS) of sputum or gastric washings in younger patients, synovial or bone biopsy for tissue PCR (GeneXpert MTB/ RIF [Cepheid, USA]) and tissue MCS. Synovium was routinely sent for histological analysis. Not all patients had results for all the abovementioned tests, therefore analysis was performed on the available results.

Plain X-rays at presentation were evaluated for radiographic features of TB. X-rays were then classified according to the system described by Shanmugasundaram ${ }^{6}$ (Figure 1a-g) for TB of the hip, and that of Kerri and Martini for TB of the knee and elbow (Table I). ${ }^{7}$ Chest X-ray was routinely performed as part of the diagnostic work-up.

Following diagnosis of MSK TB and initiation of anti-tuberculous drugs, patients were typically admitted to a long-stay facility associated with our unit. In cases where compliance was questionable due to socio-economic factors, patients remained admitted for the duration of treatment. Response to treatment was monitored with serial ESR measurements and radiographs. Duration of medical treatment was nine months, but would be prolonged if incomplete resolution of disease was noted after nine months of treatment.

Statistical analysis was performed using the StatTools tool pack in Microsoft Excel. Descriptive data was presented in the form of means and standard deviations for continuous variables, and proportions for categorical variables. Independent sample t-tests (for continuous variables), and chi-squared tests (for categorical variables) were used to assess the relationship between various independent variables. Where appropriate, statistical significance was set at $p<0.05$.

Table I: The system described by Kerri and Martini to classify TB of the knee and elbow ${ }^{7}$

\begin{tabular}{|l|l|l|}
\hline Stage & Description & \multicolumn{1}{|c|}{ Appearance } \\
\hline 1 & Normal & $\begin{array}{l}\text { Osteopaenia and soft tissue swelling with or } \\
\text { without epiphyseal hypertrophy }\end{array}$ \\
\hline 2 & Osteomyelitic & $\begin{array}{l}\text { Epihyseal or metaphyseal cysts, normal joint } \\
\text { space }\end{array}$ \\
\hline 3 & Arthritic & $\begin{array}{l}\text { Narrow joint space without gross anatomical } \\
\text { disorganisation }\end{array}$ \\
\hline 4 & Arthritic & Gross anatomical disorganisation \\
\hline
\end{tabular}


Table II: Presenting features in patients diagnosed with extra-axial MSK TB in order of frequency

\begin{tabular}{|l|c|}
\hline Presenting feature & Frequency \\
\hline Tenderness or pain & $99 \%$ \\
\hline Gait abnormalities & $77 \%$ \\
\hline Decreased range of motion & $75 \%$ \\
\hline Swelling or effusion & $66 \%$ \\
\hline TB contact (relative or person living in the same house) & $22 \%$ \\
\hline History of trauma & $14 \%$ \\
\hline Constitutional symptoms (malaise, loss of weight, loss of & $13 \%$ \\
\hline appetite) & \\
\hline Joint contracture & $8 \%$ \\
\hline HIV co-infection & $7 \%$ \\
\hline Limb length discrepancy & $4 \%$ \\
\hline
\end{tabular}

\section{Results}

All patients had symptoms or signs localised to the involved joint, such as pain, inability to bear weight, swelling, limp and deformity. The mean duration of symptoms prior to presentation was four months (one day to three years).

Seventy-six patients presented with pain and tenderness localised to an extremity. The one patient who did not have pain presented with a painless deformity of the distal tibia. Presenting features are summarised in Table II.

Of the 77 patients included, HIV status at time of presentation could be determined for 34 patients, of whom four were HIVpositive. This is $5 \%$ of the total study population and $12 \%$ of those whose results were available.

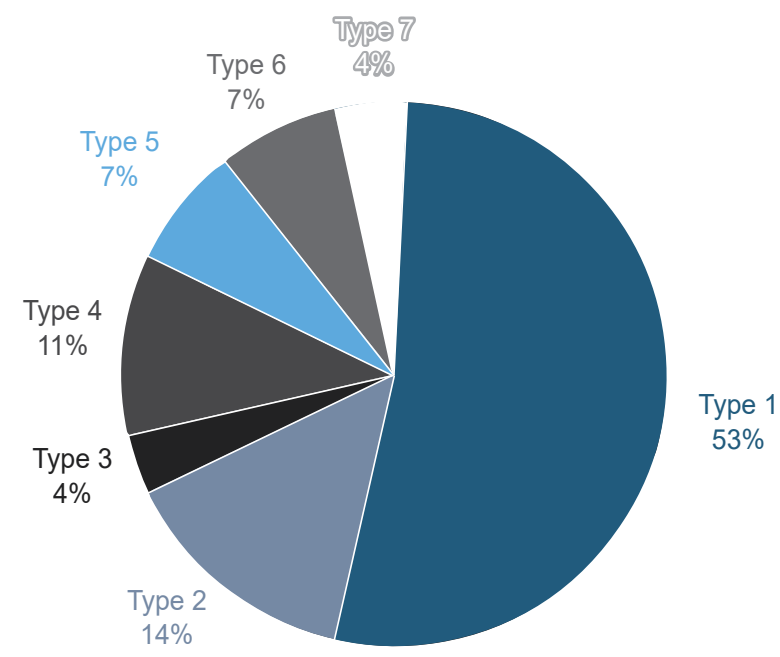

Figure 2. The frequency of radiographic type, as described by Shanmugsundaram, ${ }^{6}$ at initial presentation for patients diagnosed with tuberculosis of the hip
The hip was the most frequently involved joint $(n=29)$, followed by the knee $(n=22)$, the foot and ankle $(n=15)$, the elbow $(n=18)$ and clavicle $(n=1)$.

The results of the haematological investigations are presented in Table III. Our patients generally demonstrated a mild anaemia, thrombocytosis and elevated inflammatory markers.

Of the 29 patients diagnosed with TB of the hip, the most common radiographic presentation was a type 1 hip ('normal' hip) according to the Shanmugasundaram classification. ${ }^{6}$ The frequency of the different types of radiographic presentation for TB of the hip in our population is illustrated in Figure 2.

The most common stage at presentation for TB of the knee and elbow was stage 1 according the Kerri and Martini staging system. ${ }^{7}$ The frequency of the different stages at presentation is illustrated in Figure 3.

Chest X-ray demonstrated hilar adenopathy or other features of pulmonary TB in 22 patients out of 71 for whom these $X$-rays were available (31\%).

Of the array of diagnostic tests performed for each patient, the Mantoux skin test yielded the highest number of positive results (55/67 tested; $82 \%$ ) followed by tissue culture and/or histology (41/73 available results; $56 \%$ ), tissue PCR (39/73 tested; 53\%), tissue microscopy (33/74 available results; $45 \%$ ) and sputum/ gastric washings (11/59 available results; 19\%). Necrotising or caseating granulomas on histology were considered diagnostic of TB.

Seventy-four out of 77 patients included in the study underwent open biopsy. The most common intra-operative finding in all surgical cases was synovial hypertrophy. Frank purulence was uncommon and only seen in nine cases. The rest had either serous or straw-coloured synovial fluid. One patient underwent an open reduction of a dislocated hip at initial presentation. Three years

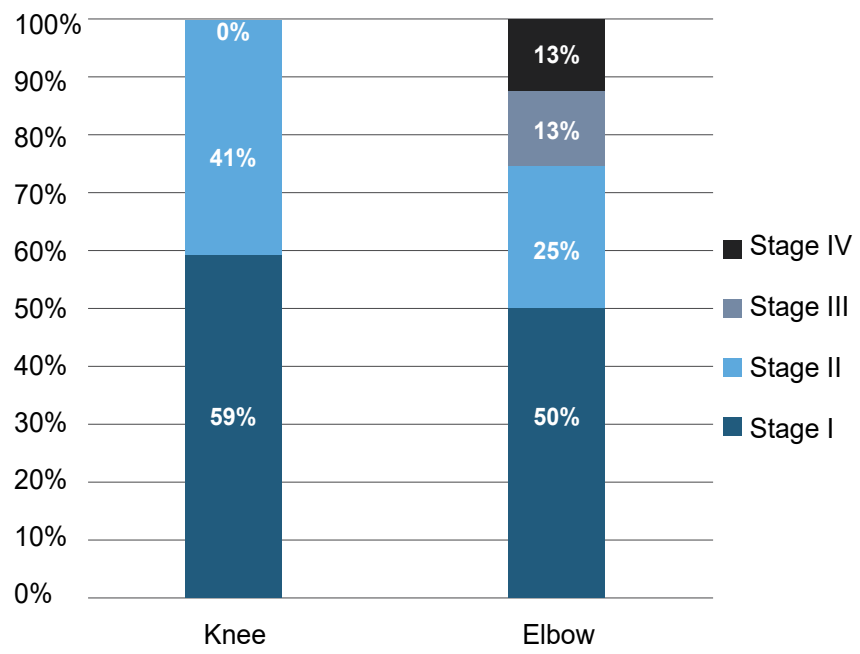

Figure 3. Frequency of radiographic stages, as described by Kerri and Martini, ${ }^{7}$ at initial presentation for patients diagnosed with tuberculosis of the knee and elbow

Table III: Haematological findings in patients diagnosed with extra-axial MSK TB

\begin{tabular}{|l|c|c|c|c|}
\hline \multicolumn{1}{|c|}{ Haematological parameter } & $\mathbf{n}^{*}$ & Normal range & Study population mean (range) & p-value \\
\hline White cell count (WCC) & 71 & $4-12 \times 10^{9} / \mathrm{L}$ & $10.61 \times 10^{9} / \mathrm{L}(4.59-30.45)$ & 0.766 \\
\hline Haemoglobin (Hb) & 60 & $11.8-14.6 \mathrm{~g} / \mathrm{dL}$ & $11.15 \mathrm{~g} / \mathrm{dL}(7-13.1)$ & 0.01 \\
\hline Platelet count (PIt) & 46 & $180-440 \times 10^{9} / \mathrm{L}$ & $458 \times 10^{9} / \mathrm{L}(192-807)$ & 0.003 \\
\hline CRP & 40 & $<10 \mathrm{mg} / \mathrm{L}$ & $29.2 \mathrm{mg} / \mathrm{L}(1-219)$ & $<0.001$ \\
\hline ESR & 67 & $0-10 \mathrm{~mm} / \mathrm{hr}$ & $42.1 \mathrm{~mm} / \mathrm{hr}(40-121)$ & $<0.001$ \\
\hline
\end{tabular}

${ }^{*} \mathrm{n}$ designates the number of patients for whom results were available for the specific blood parameter 

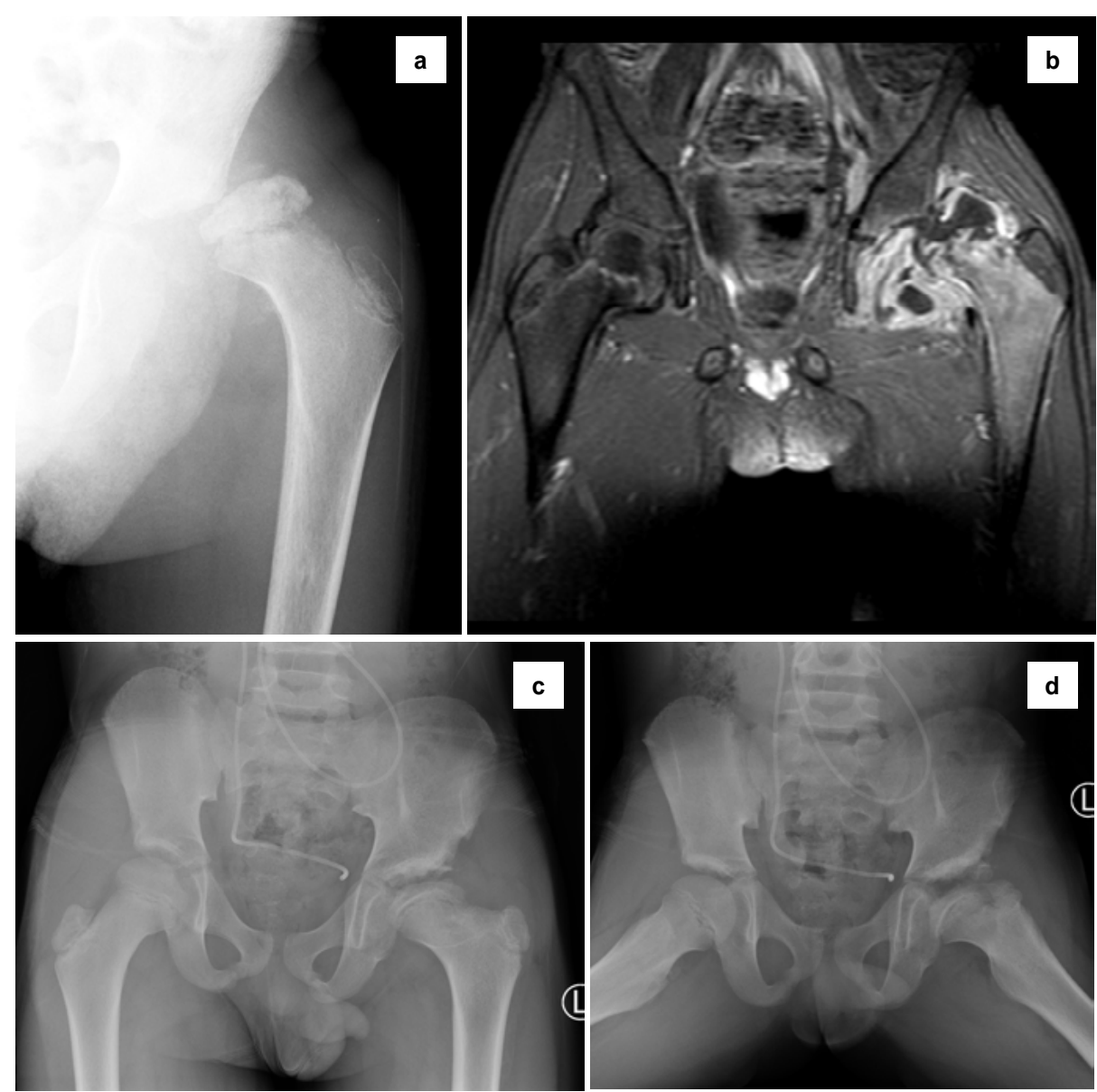

Figure 4. a) Left hip dislocation in a 7-year-old male with associated TB meningitis; b) MRI in the same patient showing large intra-articular abscess, surrounding oedema and deformity of the femoral head; (c, d) X-rays at three-year follow-up. There is residual proximal femoral deformity but a congruent joint and near normal range of motion. Note the VP-shunt in situ.
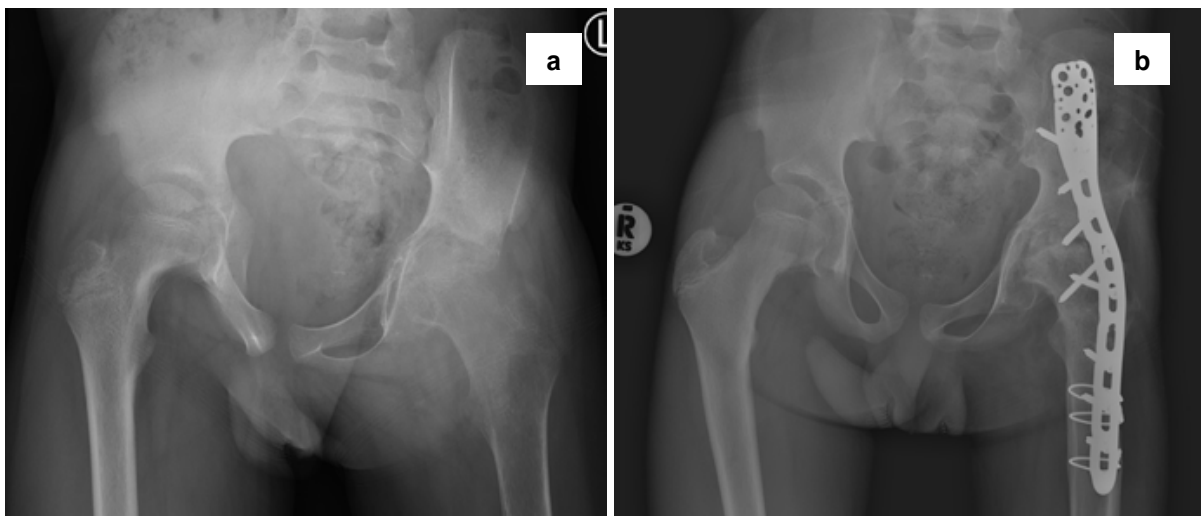

Figure 5. a) Pelvic X-ray of a 13-year-old male with a destroyed hip secondary to advanced TB; b) Hip arthrodesis was performed for persistent pain and severe deformity leading to resolution of symptoms

following the open reduction, he has near full range of motion in the hip, a $1 \mathrm{~cm}$ leg length discrepancy and a slight Trendelenburg limp (Figure 4a-d). One patient had an intralesional curettage and bone grafting of a proximal femoral lesion thought to be a unicameral bone cyst. The biopsy results revealed sensitive TB and the lesion healed with medical treatment.

Three patients received medical treatment only. Medical treatment consisted of nine months of four-drug therapy (rifampicin, isoniazid, pyrazinamide and ethambutol). Five patients demonstrated isolated INH resistance (resistance to isoniazid), which did not alter the medical treatment as per our institution's infectious
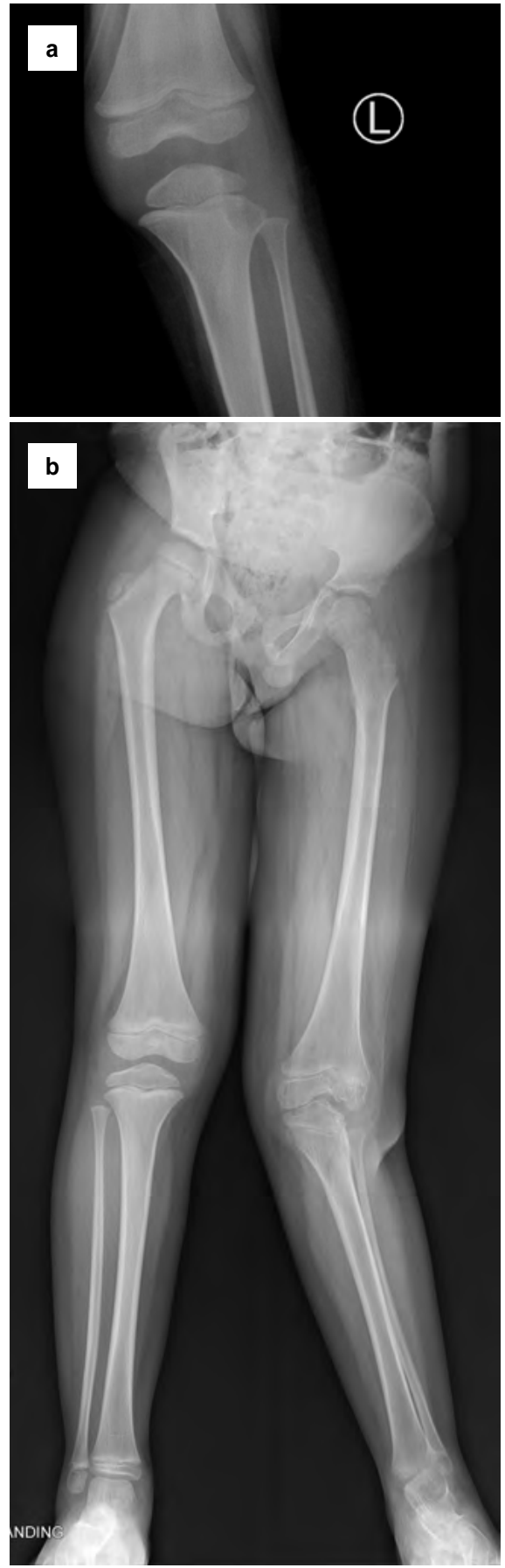

Figure 6. a) Left knee of a 5-year-old male showing a lytic lesion involving the lateral epiphysis and metaphysis of the proximal tibia; b) X-rays of the same patient after completion of medical treatment with growth arrest and progressive valgus deformity of the lower limb

diseases protocol. Compliance with medical treatment was $94 \%$. Three patients were lost to follow-up immediately following diagnosis, and two patients underwent partial treatment before defaulting.

Secondary surgery consisted of hip arthrodesis in two patients for persistent pain and severe deformity (Figure 5). One patient developed proximal tibial growth arrest and progressive valgus deformity, for which a high tibial osteotomy was performed (Figure 6). Further reconstructive surgery is planned for this patient.

No patient had persistent active disease following completion of medical treatment. Thirty patients $(39 \%)$ had some loss of range 
of motion, ranging from ankylosis to mildly decreased range, or deformity of the affected joint/limb at the final follow-up.

\section{Discussion}

TB is one of the top ten causes of death worldwide and the leading cause of mortality among HIV-positive patients. ${ }^{8}$ Extrapulmonary TB can account for up to $20 \%$ of all TB cases, with MSK TB accounting for $10-35 \%$ of these. ${ }^{9}$ The purpose of this retrospective study was to provide the most common clinical presentation of MSK TB in the paediatric patient to provide recommendations for diagnostic workup and treatment.

In our population, the most common presenting symptom was, not surprisingly, pain. This was closely followed by gait abnormalities if TB involved the lower extremity, and decreased range of motion if it involved a joint. Unlike the study by Mulleman et al. that showed that fever or weight loss was present in up to $42 \%$ of patients with spinal TB, constitutional symptoms were present in only $13 \%$ of the patients with extra-axial TB in our study. ${ }^{10}$ No other presenting symptom or sign was found to be specific to MSK TB, or to aid in differentiating MSK TB from other MSK conditions. MSK TB may, on rare occasions, present with symptoms and signs mimicking acute bacterial infection as noted by Lee et al. ${ }^{11}$ It is therefore routine practice at our institution to send samples for TB culture, microscopy and PCR in all patients that undergo biopsy for suspected acute septic arthritis or osteomyelitis.

Patients in our population were prone to thrombocytosis and anaemia with mean values being significantly different from normal values in children. The association between thrombocytosis and MSK TB has been described in TB of the spine, ${ }^{12}$ but not to our knowledge in MSK TB in children. Our patients had raised ESR and CRP values but normal WCC. The ESR is widely used as a diagnostic and prognostic tool in TB infections, ${ }^{12,13}$ and is our preferred investigation to monitor treatment response.

The mean CRP in our patients was $29.2 \mathrm{mg} / \mathrm{dL}$. Though higher than the maximum normal value of $10 \mathrm{mg} / \mathrm{dL}$, it was low compared to the mean CRP in patients presenting with acute pyogenic osteomyelitis in our population, which is $223.6 \mathrm{mg} / \mathrm{dL} .{ }^{14}$ This finding may be helpful in distinguishing pyogenic infections from tuberculous infections, as also proven by Niu et al. in the setting of pneumonia. ${ }^{15}$

Of the available diagnostic tests specific for Mycobacterium tuberculosis (MTB), the Mantoux skin test had the highest positive rate in our study population ( $82 \%$ ) followed by tissue culture and/or histology (56\%), tissue PCR (53\%), tissue microscopy (45\%), and sputum/gastric washings (19\%). A positive Mantoux test should be interpreted with caution as false positives may occur in the setting of prior BCG vaccination, which forms part of our national vaccination programme. Children who have latent TB, but no active disease, may also demonstrate a positive Mantoux test, and the test may be falsely negative in patients who are immunocompromised due to, for example, HIV infection.

Tissue PCR has been shown to have a sensitivity and specificity for TB of $92.3 \%$ and $99.1 \%$ respectively in a series including spinal TB from our institution. ${ }^{16}$ The reason for the lower diagnostic sensitivity in our series may be the quality of our samples and the absence of frank pus in most of our patients. Lee et al. demonstrated a sensitivity of $17.6 \%$ for PCR in their study and found the sensitivity to be much improved if necrotic tissue was present. ${ }^{17}$

Only four out of 77 patients (5\%) were known to be HIV-positive at time of presentation. Results could only be found for 34 of our patients but even then, the incidence is $12 \%$. In a study examining extra-axial MSK TB in children, Firth et al. reported a prevalence of $33.3 \%$ of HIV co-infection. ${ }^{18}$ The reason for the low HIV co-infection rate in our patients remains elusive, but TB is endemic in our region, as is demonstrated by the four-times higher number of cases in our study than in that by Firth et al. ${ }^{18}$ over the same time period, and may be ascribed to different epidemiology and disease trends in our population.

The systems described by Shanmugasundaram ${ }^{6}$ and Kerri and Martini ${ }^{7}$ for MSK TB are helpful in recognising typical radiological presentations. The most common radiographic presentation of TB of the hip in our population was type 1, which is 'normal', followed by type 2 , the travelling type. It should be noted that type 1 hips are seldom completely normal and may demonstrate generalised osteopaenia and/or displacement of the soft-tissue planes, if an effusion is present. These results were similar to results from a retrospective study of 27 patients by Agarwal et al. ${ }^{19}$ All seven radiographic stages were represented in our population, even though there was only one case each of type 3 (dislocating) and type 7 (pestle and mortar). This differs from other studies where certain types were not seen. ${ }^{19,20}$ In the case of knee-and-elbow TB, the most common stage at presentation was stage 1.

The treatment of MSK TB is primarily medical and, depending on the stage of disease at presentation, surgery may be avoided completely. ${ }^{21}$ MSK TB can usually be diagnosed clinically, particularly in areas where the disease is endemic. Despite this, biopsy is recommended, if not mandatory, to exclude other pathology as well as to determine sensitivity due to the rise in drug resistant TB. ${ }^{22}$

All but three patients in our study underwent open biopsy in order to confirm the diagnosis. In cases where a large cold abscess was present, this was drained and the joint debrided. No patient required a synovectomy to control active disease, although this has been reported in other literature. ${ }^{21}$

All our patients that were followed up to completion of treatment had complete resolution of active disease, as demonstrated by a healed lesion on X-ray and normal ESR levels. Compliance with the intensive and prolonged medical treatment regimen is paramount to treatment success and avoidance of recurrence or resistance. ${ }^{23}$ We had a $94 \%$ compliance rate as many of our patients were hospitalised for the duration of treatment. A recent study looking at factors influencing adherence to TB treatment by Gebreweld et al. showed that short distances to health facilities, good communication with and health education of families, and strengthening of social structures can enable better adherence to TB treatment. ${ }^{24}$ These factors should be considered by physicians lacking the facilities or resources to admit patients to the inpatient ward for the duration of TB treatment, to improve compliance.

Residual deformity and joint stiffness are common following successful treatment of MSK TB. Only three of our patients have required orthopaedic surgery to address residual pain and deformity, but we anticipate that many will require surgery in future for early onset osteoarthritis of the involved joint.

There are several limitations to our study. As it is a retrospective study, it is subject to biases inherent to its design, such as selection bias. Investigations were not necessarily performed systematically in all patients, and treatment initiation was at the discretion of the treating physician. Our follow-up was short, and we cannot comment on the long-term outcomes of patients with MSK TB. Despite these limitations, the major strengths of this paper are the large number of patients and data points collected for each patient in the sample. This allowed us to investigate patterns in laboratory values, imaging findings, demographics, treatments and outcomes, and provide a comprehensive review of how a patient with MSK TB might be diagnosed and treated appropriately. 


\section{Conclusion}

MSK TB should always be considered as part of the differential for bone and joint pain in children, especially in endemic areas. Certain clinical, radiological and haematological patterns are typical, though not diagnostic; diagnostic testing should include a variety of modalities including an open biopsy, as no individual test is $100 \%$ sensitive or specific. Initial treatment is medical and will lead to resolution of disease in nearly all patients that complete the full treatment course. Orthopaedic surgery may be indicated for residual deformity or disability once medical treatment is complete.

\section{Ethics statement}

Approval as obtained from the institutional Human Research Ethics Committee (HREC Ref 183/2019).

The authors declare that this submission is in accordance with the principles laid down by the Responsible Research Publication Position Statements as developed at the 2nd World Conference on Research Integrity in Singapore, 2010.

All procedures were in accordance with the ethical standards of the responsible committee on human experimentation (institutional and national) and with the Helsink Declaration of 1975 , as revised in 2008. Informed written consent was not obtained from all patients for being included in the study.

\section{Declaration}

The authors declare authorship of this article and that they have followed sound scientific research practice. This research is original and does not transgress plagiarism policies.

\section{Author contributions}

SV: Study design, data capturing and analysis, preparation of manuscript

$\mathrm{AH}$ : Study design, data capturing and analysis, editing of manuscript, supervision

\section{References}

1. United States Centers for Disease Control and Prevention. Reported Tuberculosis in the United States, 2016. https://www.cdc.gov/tb/statistics/ reports/2016/pdfs/2016_Surveillance_FullReport.pdf.

2. Held MFG, Hoppe S, Laubscher M, et al. Epidemiology of musculoskeletal tuberculosis in an area with high disease prevalence. Asian Spine J. 2017;11(3):405-11.

3. Kramer N, Rosenstein ED. Rheumatologic manifestations of tuberculosis. Bull Rheum Dis. 1997 May;46(3):5-8.

4. Vohra R, Kang HS, Dogra S, Saggar RR, Sharma R. Tuberculous osteomyelitis. J Bone Joint Surg (Br). 1997;79(4):562.

5. Rasool MN. Tuberculosis-the masquerader of bone lesions in children. SA Orthop J. 2009;8(1):21-25.

6. Shanmugasundaram TK. Bone and Joint Tuberculosis. Madras: Kothandaram and Co; 1983.

7. Kerri O, Martini M. Tuberculosis of the knee. Int Orthop. 1985;9(3):153-57. https://www.ncbi.nlm.nih.gov/pubmed/4077334.

8. WHO Media Center. Tuberculosis. WHO. 2018. Available from: http://www.who int/mediacentre/factsheets/fs104/en/.

9. Held M, Bruins MF, Castelein S, et al. A neglected infection in literature: childhood musculoskeletal tuberculosis - A bibliometric analysis of the most influential papers. Int J Mycobacteriology. 2017;6(3):229-38.

10. Mulleman D, Mammou S, Griffoul I, et al. Characteristics of patients with spinal tuberculosis in a French teaching hospital. Joint Bone Spine. 2006 Jul;73(4):424-27.

11. Lee AS, Campbell JAB, Hoffman EB. Tuberculosis of the knee in children. $J$ Bone Joint Surg (Br). 1995;77-B:313-18.

12. Daniel K, Dunn R. Comparison of platelet count in tuberculosis spine to other spine pathology. Europ Spine J. 2013;22(12):2810-14.

13. Tengku Muzaffar TMS, Shaifuzain AR, Imran Y, Noor Haslina MN. Hematological changes in tuberculous spondylitis patients at the Hospital Universiti Sains Malaysia. Southeast Asian J Trop Med Public Health. 2008;39(4):686-89.

14. Horn A, Wever S, Hoffman EB. Complications following acute severe haematogenous osteomyelitis of the long bones in children. SA Orthop J. 2019;18(3):23-29.

15. Niu W-Y, Wan Y-G, Li M-Y, et al. The diagnostic value of serum procalcitonin, IL-10 and C-reactive protein in community acquired pneumonia and tuberculosis. Eur Rev Med Pharmacol Sci. 2013;17:3329-33.
16. Held M, Laubscher M, Workman L, et al. Diagnostic accuracy of GeneXpert MTB/RIF in musculoskeletal tuberculosis: High sensitivity in tissue samples of HIV-infected and HIV-uninfected patients. S Afr Med J. 2017;107(10):854-58.

17. Lee YJ, Kim S, Kang Y, et al. Does polymerase chain reaction of tissue specimens aid in the diagnosis of tuberculosis? J Pathol Transl Med. 2016;50(6):451-58. https://www.ncbi.nlm.nih.gov/pmc/articles/PMC5122730/.

18. Firth GB, Lescheid J, Camacho M, et al. Extraspinal osteoarticular multidrug-resistant tuberculosis in children: A case series. S Afr Med J. 2017;107(11):983-86. https://doi.org/10.7196/SAMJ.2017.v107i11.12577.

19. Agarwal A, Suri T, Verma I, et al. Tuberculosis of the hip in children: a retrospective analysis of 27 patients. Indian J Orthop. 2014;48(5):463-69. https://www.ncbi.nlm.nih.gov/pmc/articles/PMC4175859/\#ref8.

20. Campbell JAB, Hoffman EB. Tuberculosis of the hip in children. J Bone Joint Surg (Br). 1995;77-B(2):319-26.

21. Tuli SM. General principles of osteoarticular tuberculosis. Clin Orthop Relat Res. 2002;398(1):11-19.

22. Dunn R. The medical management of spinal tuberculosis. SA Orthop J. 2010;9(1):37-41.

23. Dick J, Lombard C. Shared vision - a health education project designed to enhance adherence to anti-tuberculosis treatment. Int J Tuberc Lung Dis. 1997 Apr;1(2):181-86.

24. Gebreweld FH, Kifle MM, Gebremicheal FE, et al. Factors influencing adherence to tuberculosis treatment in Asmara, Eritrea: a qualitative study. J Health Popul Nutr. 2018;37:1. https://www.ncbi.nlm.nih.gov/pmc/articles/ PMC5756387/. 